\title{
The Time to Detect Targets at Inhibited and Noninhibited Locations: Preliminary Evidence for Attentional Momentum
}

\author{
Jay Pratt \\ University of Toronto
}

\author{
Thomas M. Spalek \\ University of Toronto at Scarborough
}

\author{
Frederick Bradshaw \\ Washington University
}

\begin{abstract}
Inhibition of return refers to the finding that response latencies are longer for targets appearing at previously attended (cued) locations than at novel (uncued) locations. The present research was designed to examine the pattern of detection latencies that occurred for targets appearing at various uncued locations. The first 2 experiments showed that responses were fastest when the target occurred at a location directly opposite the cue. Experiment 3 showed that latencies were related to the angle between the target and the direction in which attention was being oriented. Experiments 4 and 5 showed that manipulating the direction of attentional orientation affected inhibition of return. Overall, the results suggest that inhibition of return may be due to the difference between orienting attention to locations along the path of attention versus orienting attention to those off the path of attention.
\end{abstract}

The time to detect a target appearing at a peripheral location is influenced by several factors, including the intensity of the target stimulus (e.g., Hawkins, Shafto, \& Richardson, 1988) and the eccentricity of the peripheral target location (e.g., LaBerge \& Brown, 1986). Another factor that has been found to have a profound influence on the time to detect a target is the manner in which attention is allocated at the time the target is presented. Posner (1980) found that response latencies to detect a peripheral target were reduced when attention was allocated to the target location just before the onset of the target. This occurred despite the fact that the observers did not move their gaze from a central fixation point and regardless of whether attention was involuntarily oriented (through the use of an abrupt-onset peripheral cue) or voluntarily oriented to a location (with a central cue indicating to which location attention should be directed). This facilitation of detection responses at attended locations has become known as "attentional cuing."

While examining the effects of attentional cuing, Posner

Jay Pratt, Department of Psychology, University of Toronto, Toronto, Ontario, Canada; Thomas M. Spalek, Department of Psychology, University of Toronto at Scarborough, Ontario, Canada; Frederick Bradshaw, Department of Psychology, Washington University.

Frederick Bradshaw is now at the University of Richmond School of Law.

This research was supported by Natural Sciences and Engineering Research Council of Canada Awards 0194537 and 0196162. We would like to thank three anonymous reviewers and especially Hermann Müller for helpful comments on an earlier version of this article.

Correspondence concerning this article should be addressed to Jay Pratt, Department of Psychology, University of Toronto, 100 St. George Street, Toronto, Ontario, Canada M5S 3G31. Electronic mail may be sent to pratt@psych.utoronto.ca. and Cohen (1984) discovered a phenomenon in which responses to detect the appearance of a target were inhibited. Specifically, Posner and Cohen found that if a relatively long delay period intervened between the presentation of a peripheral cue and the presentation of a target (a delay of more than $300 \mathrm{~ms}$ ), participants were slower to detect targets at the cued location. Posner and Cohen coined the term inhibition of return, referring to their notion that attention was inhibited in returning to previously attended locations. They reasoned that during the delay, attention had moved from the cued location to the central fixation location, resulting in the cued location's becoming the previously attended location. Assuming that an efficient attentional search strategy would involve avoiding attending alreadyattended locations, Posner and Cohen suggested that inhibition of return might reflect a mechanism that improves the efficiency of attentional searches by biasing attention toward novel locations and away from previously attended locations.

Since the initial finding of inhibition of return by Posner and Cohen (1984), the inhibitory phenomenon has been the focus of a great deal of research interest. From this research a good deal of information has been gained about inhibition of return. It is known that inhibition of return occurs when attention is involuntarily allocated to a peripheral location but not when attention is voluntarily oriented with a central cue (Posner \& Cohen, 1984). The exception to this is that inhibited detection responses are found if the voluntary orientation of attention coincides with either an overt eye movement or the preparation of an eye movement (Rafal, Calabresi, Brennan, \& Sciolto, 1989). Inhibition of return has also been found with various types of responses, including simple manual keypresses (e.g., Maylor, 1985; Reuter-Lorenz, Jha, \& Rosenquist, 1996), choice manual keypresses (e.g., Maylor, 1985), and eye move- 
ment responses (e.g., Abrams \& Dobkin, 1994; Vaughan, 1984).

Over the past several years, inhibition of return has proved to be a robust inhibitory effect. Work by Tipper and others has shown that the inhibition tends to stay with a previously attended object even if that object moves away from the location where it was cued (e.g., Abrams \& Dobkin, 1994; Gibson \& Egeth, 1994; Tipper, Driver, \& Weaver, 1991; Tipper, Weaver, Jerreat, \& Burak, 1994; but see Müller \& von Mühlenen, 1996). It also appears that inhibition of return may affect more than one previously attended location (e.g., Abrams \& Pratt, 1996; Danziger, Kingstone, \& Snyder, 1998; Tipper, Weaver, \& Watson, 1996; Wright \& Richard, 1996), although, depending on the circumstances, only the most recently attended location may yield inhibited detection responses (Abrams \& Pratt, 1996; Pratt \& Abrams, 1995). Despite some early evidence to the contrary (Tanaka \& Shimojo, 1996; Terry, Valdes, \& Neill, 1994), there now appears to be growing support for the finding that inhibition of return may occur not only for responses based on the detection of a target but also for responses that are based on the identity of the target stimulus (Chasteen \& Pratt, 1999; Lupiáñez, Milán, Tornay, Madrid, \& Tudela, 1997; Pratt, 1995; Pratt \& Abrams, 1999; Pratt, Kingstone, \& Khoe, 1997).

Although the plethora of research concerning inhibition of return has provided several important insights into the inhibitory effect, the research up to this moment has almost exclusively focused on what happens at the previously attended location. The approach taken in the present research was a little different in that the present experiments were designed to examine what happens at the nonattended locations when one location was previously attended. Imagine the following situation: One is presented with a display consisting of multiple potential target locations, and the target is equally likely to appear at any one of those locations. One's attention is oriented to one of those locations and then is reoriented back to where one is fixating. At this point, inhibition of return should act on the previously attended location, so it will be more difficult to return one's attention back to that location. However, because the target is equally likely at all four of the locations, it is more likely to occur at an unattended (i.e., uncued) location than at the single previously attended (i.e., cued) location. Thus, understanding what factors influence the detection of targets at various uncued locations is at least as important as determining what factors affect target detection at cued locations. Examining uncued locations in an inhibition-of-return paradigm is especially important when one considers that it is a relatively rare attentional search in which one must detect an object at one of two locations (one previously attended and one unattended). More than likely, people conduct visual searches in environments where there are multiple locations in which the target object might occur. We designed the present research to gain some insight into the mechanisms that underlie the detection of targets at unattended locations when attention has already been previously allocated to a single location.

\section{Response Latencies at Uncued Locations}

To help clarify the alternative hypotheses for what might happen at uncued locations, we will use a basic hypothetical paradigm. This paradigm consists of a central fixation point surrounded by four potential target locations at $90^{\circ}$ angles to each other (i.e., the four locations form a cross with the fixation at the intersection). Assume that a cue appeared at the top location, followed by a cue at the fixation point (to reorient attention back to fixation). The top location is the inhibited location because it was previously attended (i.e., the cued location), and the left, right, and bottom locations are the unattended locations (i.e., the uncued locations). The target is equally likely to occur at any of the four locations, and the participants are asked to make a simple keypress as soon as they notice the appearance of the target. With this hypothetical paradigm, it is possible to examine the various potential hypotheses and what pattern of results each will predict.

The simplest hypothesis may be called the "inhibited location hypothesis." This hypothesis suggests that only the previously attended location will be inhibited and that the time to detect a target at any of the other three locations will be approximately the same. Evidence contradicting this hypothesis and in support of the other hypothesis is given below.

Another hypothesis may be called the "spreading inhibition hypothesis." This hypothesis suggests that inhibition will spread from the inhibited location, gradually weakening as the distance from the inhibited location increases. The prediction from this hypothesis would be that the detection responses would be slowest at the top location (the inhibited location), next slowest at the left and right locations (closest to the inhibited location), and fastest at the bottom location (farthest from the inhibited location). There is some evidence to suggest that inhibition may spread from an inhibited location to other locations and that the spread of inhibition is affected by distance. For example, Berlucchi, Tassinari, Marzi, and Di Stefano (1989) used a display consisting of four light-emitting diodes (LEDs), two on each side of a central fixation point. One LED was briefly illuminated as the cue, and this was followed by illumination of the same or a different LED as the target. As expected, Berlucchi et al. found that response latencies were slowest when the same LED was used for the cue and the target. However, they also found evidence for inhibition of return at the uncued LED on the same side of fixation as the cued LED. Presumably, inhibition spread from the cued location to the nearby uncued location. Similar patterns of results were found by Maylor and Hockey (1985), Tassinari, Aglioti, Chelazzi, Marzi, and Berlucchi (1987), and Tassinari and Campara (1996).

A third alternative may be called the "inhibited hemifield hypothesis." This hypothesis suggests that inhibition will spread over the hemifield in which the cued location occurred. Thus, this hypothesis is like a limited version of the spreading inhibition hypothesis, because inhibition would 
spread from an inhibited location until it reached either a vertical or horizontal meridian (and then being drastically reduced on the other side of the meridian). The prediction from this hypothesis is that targets will be detected the slowest at the cued location and the next slowest at the left and right locations (because each of those locations shares one hemifield with the cued location) and that response latencies will be the fastest at the bottom location (which does not share a hemifield with the cued location). This hypothesis stems from findings that attention tends to spread within a quadrant (as defined by vertical and horizontal meridians) and has difficulty in crossing the meridians (e.g., Hughes \& Zimba, 1985, 1987; Rizzolatti, Riggio, Dascola, \& Umilta, 1987). More directly related is the conclusion of Tassinari et al. (1987) that the vertical and horizontal meridians provide a similar barrier to the spread of inhibition outward from a cued location.

The final hypothesis may be called the "attentional momentum hypothesis." This hypothesis suggests that attention tends to continue in the direction in which it last traveled. The assumption is that attention has something like momentum associated with it that allows it to be oriented to locations along the direction of orientation faster than to locations that require a change in the direction of orientation. The prediction of this hypothesis revolves around the direction of the movement of attention before the target presentation. In the case of the present hypothetical experiment, the movement of attention would be down from the top cued location to the central fixation cue. Thus, the attentional momentum hypothesis would predict that response latencies would be slowest at the cued location $\left(180^{\circ}\right.$ off the direction of attention), next slowest at the left and right locations $\left(90^{\circ}\right.$ off the path of attention), and fastest at the bottom location (along the path of attention). Note that this hypothesis does not suggest that attention actually overshoots the fixation location because it has momentum. Rather, we suggest that attention momentum may best be thought of as the bias for attention to continue moving in the direction in which it most recently traveled.

This momentum view is not a completely novel one. Corbetta, Miezin, Shulman, and Petersen (1993), in a positron emission tomography study, used the term momentum effect to describe the difference in reaction times (RTs) between validly cued and invalidly cued locations in a shifting attention task. In their task, however, this was a completely endogenous effect because $80 \%$ of their trials were validly cued. Thus, as Corbetta et al. noted, the momentum effect was probably due to the predictability of the target location and the instructions that the participants should shift their attention to that location. In the present experiment, however, we were interested in looking at more involuntary attentional shifts, and to do so, we used exogenous cues that were completely nonpredictive of target locations.

Further evidence for the attentional momentum hypothesis comes from a variety of indirect sources. First, there is evidence to suggest that attention tends to travel in an analog manner and that locations in the path of this movement are attended and locations off the path are not attended (e.g.,
O’Donnell \& Pratt, 1996; Pratt \& Abrams, 1994; Shulman, Remington, \& McLean, 1979; Tipper, Lortie, \& Baylis, 1992). For example, O'Donnell and Pratt found that cuing a more eccentric location yielded inhibition at a less eccentric target location only if the less eccentric target location was along the path that attention traveled to reach the cued location. There is also evidence for momentumlike effects in other cognitive domains, which has been termed representational momentum (for a detailed review of this work, see Hubbard, 1995). This work has shown that observers tend to recall the locations of objects not at the last location where they were presented but at locations consistent with the continued motion of the objects. Thus, the representation of the object appeared to be affected by the object's apparent momentum (e.g., Freyed \& Finke, 1984, 1985).

Although several researchers have argued for attention moving in a spotlightlike manner (e.g., Shulman et al., 1979), other researchers have argued against such a notion (e.g., Kwak, Dagenbach, \& Egeth, 1991; Sagi \& Julesz, 1985). These researchers claim that attention moves in a more abrupt, time-independent/noninertial manner. For example, Kwak et al. used a same-different letter (T vs. L) discrimination task and showed that the distance between the two letters to be discriminated made no difference in the time needed to make the discrimination. This was despite the fact that there was evidence that the items were being processed serially. Thus, there is conflicting evidence about the exact manner in which attention is oriented to locations in the visual field. However, there appears to be sufficient evidence to suggest that the notion of attentional momentum in inhibition of return should not be dismissed outright and should be examined as one possibility for the effect.

It is apparent that in the case of the aforementioned hypothetical experiment, the latter three hypotheses (i.e., spreading inhibition, inhibited hemifield, and attentional momentum) all make the same prediction for the pattern of detection latencies (but for different reasons). We made our first experiment in the present research similar to the hypothetical experiment in order to determine whether the inhibited location hypothesis or the three later hypotheses best predicted the pattern of response latencies at the uncued locations. From the first experiment the inhibited location hypothesis was eliminated, and three subsequent experiments were conducted to determine which of the remaining three hypotheses provided the best account of the data. The results from Experiment 2 eliminated the spreading inhibition hypothesis, whereas the results from Experiments 3 and 4 were inconsistent with the inhibited hemifield hypothesis and offer support for the attentional momentum hypothesis.

\section{Experiment 1}

The purpose of the first experiment was to examine whether the time to detect the appearance of a target differed between uncued locations depending on the location of the cue. To do this, participants were presented with a display consisting of a central fixation point and four peripheral target locations (indicated by placeholders). The four target 
locations were spaced equally around the fixation location, with two being aligned along the horizontal meridian and two being aligned along the vertical meridian. During each trial, the participant's attention was oriented to one of the peripheral locations and then back to the fixation location through the abrupt presentation of cues. After the fixation cue, a target appeared in one of the four peripheral locations. Thus, on each trial the target could occur at either the cued location (the previously attended location) or one of the three uncued locations (unattended locations).

Our major prediction was that the time to detect a target would be slower when the target occurred at a cued location than when it occurred at any of the three uncued locations. This would be the typical inhibition-of-return effect, and there is considerable evidence to indicate that cuing one of four locations will result in inhibited detection responses at the cued location (e.g., Tipper et al., 1994). However, the focus of the present study was not the inhibited responses to targets at the cued location but the examination of differences in RT between responses to targets presented at the various uncued locations. As mentioned earlier, there is evidence to suggest that there may be RT differences for targets presented at the different uncued locations. We conducted the first experiment to determine whether any such differences between uncued locations might be found using a paradigm that consisted of one cued location and three uncued locations, all at equal eccentricities from a central fixation point.

\section{Method}

Participants. Twelve undergraduate students from the University of Toronto participated in the study for course credit. Each student participated in a single session that lasted approximately $30 \mathrm{~min}$.

Apparatus and procedure. Participants were seated directly in front of a computer monitor with a computer keyboard located so that they could easily press any of the keys. The trial sequence is shown in Figure 1. At the start of each trial, a display consisting of a central fixation point and four target locations (indicated by placeholder boxes) was presented for $1,500 \mathrm{~ms}$. The four placeholder boxes were aligned with two along the vertical meridian and two along the horizontal meridian. Thus, the possible target locations were to the left, right, above, and below the fixation point. The center of each placeholder was located $6^{\circ}$ of visual angle from the fixation point, and the box itself subtended $1^{\circ}$. Participants were instructed to remain fixated on the fixation point throughout each trial.

After the initial display, a cue was presented (a filled-in dot) in one of the placeholder boxes for $300 \mathrm{~ms}$. The abrupt onset of the cue was assumed to capture attention at that peripheral location (e.g., Yantis \& Jonides, 1990). After a 200-ms delay, another cue was presented at the fixation point for $300 \mathrm{~ms}$. After the onset of the fixation cue, there was a delay of $150 \mathrm{~ms}$. After this final delay, the target (a filled-in square) appeared in one of the four possible target locations, and participants were instructed to press the spacebar as soon as they detected the appearance of the target. The target remained on until the participant responded or 1,000 ms elapsed from the onset of the target. If the participant made an error in responding (the RT was less than $100 \mathrm{~ms}$ or more than $1,000 \mathrm{~ms}$ ), a brief error tone was presented. To reduce the number of anticipa-

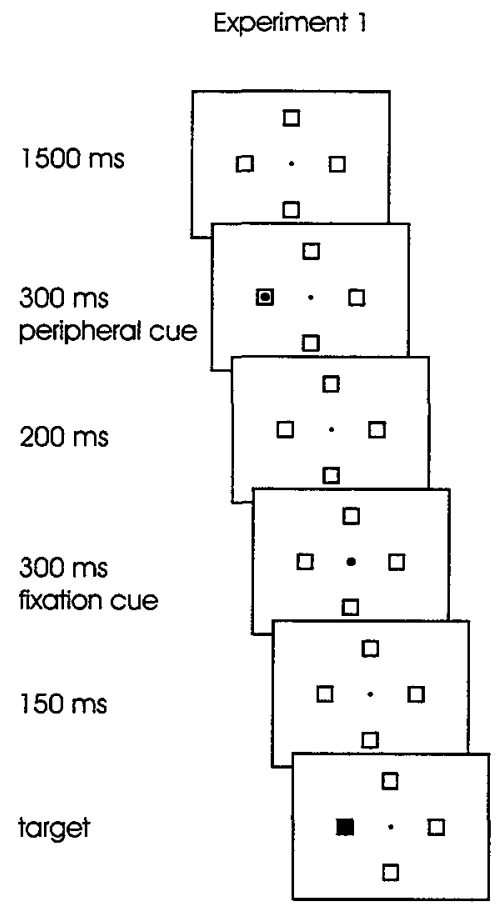

Experiment 2

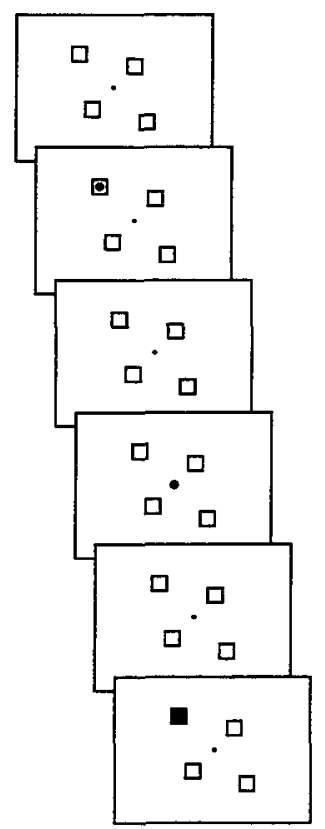

Experiment 3

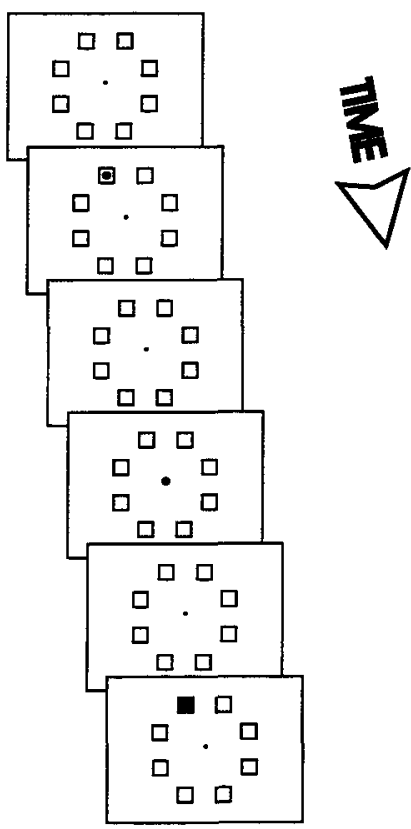

Figure 1. The trial sequences used in Experiments 1,2, and 3. The placeholders and stimuli are not drawn to scale. In all of the experiments, the peripheral cues and targets were equally likely to appear at any of the locations. 
tory responses, catch trials in which no target stimulus was presented occurred randomly throughout the experiment. The intertrial interval was $1,500 \mathrm{~ms}$.

Design. The experiment consisted of a total of 320 trials per participant, 64 trials in each of four different conditions. One condition was the cued condition, in which the target appeared in the same location as the previously presented cue. The opposite condition occurred when the target appeared in the uncued location directly opposite the location where the cue appeared. The left condition occurred when the target appeared in the uncued location situated $90^{\circ}$ to the left of the cued location (when facing the cued location). The right condition was the same as the left condition except the target location was situated $90^{\circ}$ to the right of the cued location. Finally, catch trials occurred on $20 \%$ of the trials, and on these trials no target was presented and participants were instructed to withhold making responses. Pressing any key in a catch trial resulted in an error. The cue was equally likely to appear at any of the four locations, and the conditions were completely randomized across the experiment.

\section{Results and Discussion}

The mean RTs from the correct trials appear in Figure 2. The mean RTs were analyzed with a one-way analysis of variance (ANOVA) using the four conditions (cued, opposite, left, and right). Overall, there was a main effect for conditions, $F(3,33)=31, p<.00001$, with responses in the cued condition the slowest $(402 \mathrm{~ms})$, responses in the opposite condition the fastest ( $353 \mathrm{~ms})$, and responses in the left and right conditions falling in between $(367 \mathrm{~ms}$ and 363 $\mathrm{ms}$, respectively). The same analysis was conducted on the percentage of trials in which an error occurred, and no main effect for condition was found $(F<1)$. The percentages of error trials are presented in Table 1.

To further examine the data, we performed separate paired $t$ tests on selected pairs of conditions. The first such analysis was conducted on the left and right conditions because all of the hypotheses (i.e., location inhibition, spreading inhibition, inhibited hemifield, and attentional momentum) predict that no differences should be found for locations that (a) are

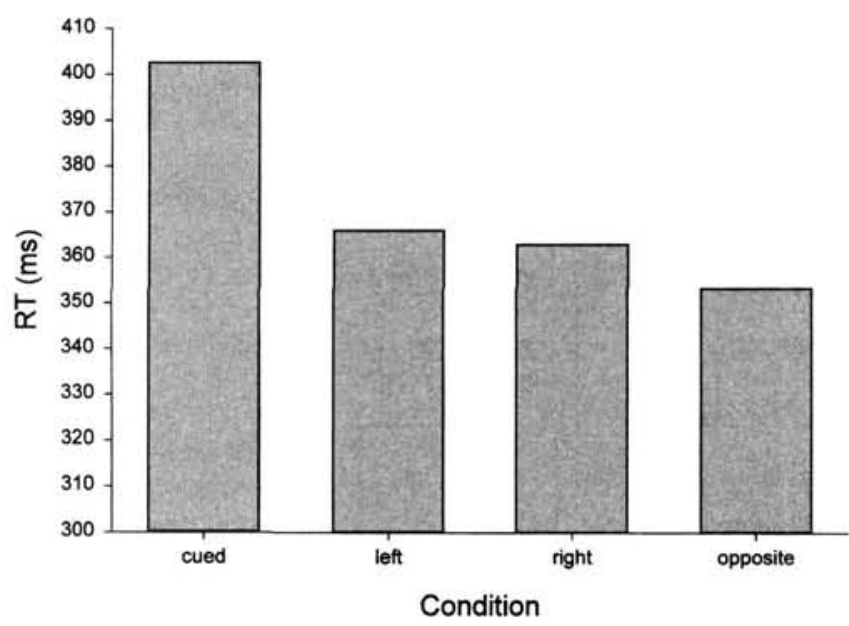

Figure 2. The mean reaction times (RTs) for the correct trials from Experiment 1.
Table 1

Percentages of Error Trials in Experiments 1-4

\begin{tabular}{lc}
\hline Experiment and conditions & Error trials (\%) \\
\hline Experiment 1 & \\
Cued & 3.6 \\
Left & 5.2 \\
Right & 4.1 \\
Opposite & 5.7 \\
Catch trials & 2.6 \\
Experiment 2 & 4.8 \\
Cued & 3.5 \\
Short opposite & 3.1 \\
Long opposite & 5.1 \\
Orthogonal & 3.3 \\
Catch trials & \\
Experiment 3 & 4.2 \\
Cued & 5.0 \\
Opposite & 3.2 \\
0-hemifield, 1-adjacent & 4.5 \\
1-hemifield, 1-adjacent & 4.6 \\
1-hemifield, 2-adjacent & 4.1 \\
1-hemifield, 3-adjacent & 3.9 \\
2-hemifield, 3-adjacent & 2.9 \\
Catch trials & \\
Experiment 4 & 5.3 \\
Cued, same side & 5.8 \\
Uncued, same side & 6.0 \\
Cued, opposite side & 5.3 \\
Uncued, opposite side & 9.8 \\
Experiment 5 & 8.6 \\
Cued, same side & 8.9 \\
Uncued, same side & 7.9 \\
Cued, opposite side & \\
Uncued, opposite side & \\
\hline &
\end{tabular}

equally distant from the cued location, (b) share one hemifield with the cued location, and (c) are equally eccentric from the path of attention. The left and right conditions fit all of the above criteria, and in fact, no differences were found between the two conditions, $t(11)<$ $1.6, p>$.14. To simplify the following analyses, we combined the data from the left and right conditions into a mean RT. This was called the "orthogonal condition."

The presence of inhibition of return in the present experiment was confirmed by separate $t$ tests in which the cued location was found to be slower than both the orthogonal condition, $t(11)=3.7, p<.004$, and the opposite condition, $t(11)=6.8, p<.00005$. Importantly, responses in the opposite condition were found to be faster than responses in the orthogonal condition, $t(11)=2.2, p<$ .05 . This indicates that cuing one of four potential target locations did not result in equal RTs for targets that appeared at the three uncued locations. Thus, it does not appear that inhibition of return affected only the responses made to targets at cued locations. Rather, the results from the present experiment indicate that the latency to detect a target at an uncued location depended on the relationship between the cued location and the target location. Specifically, responses to targets were fastest when the target occurred in the location directly opposite the cued location rather than when the target occurred in a location orthogonal to the cued location. 
In the present experiment, the opposite location differed from the orthogonal locations in three important aspects. First, the opposite location was much farther away from the cued location than were the two orthogonal locations. Thus, the difference between the RTs might have been due to a spreading of inhibition from the cued location, with the closer locations receiving more of the spreading inhibition than the opposite location. In other words, the eccentricity of the locations might have been the cause for the different uncued RTs.

The second aspect in which the opposite location differed from the orthogonal locations was that the orthogonal locations shared a hemifield of the visual field with the cued location, whereas the opposite location did not. As noted earlier, there is evidence to suggest that attention tends to spread within a hemifield after a cue. Because inhibition of return is typically thought of as an attentional phenomenon, it is reasonable to suspect that inhibition might also spread over a hemifield after a cue. Note that this assumes that the meridians do not act as absolute boundaries to the spread of attention but rather that there is a significant reduction in the spread of attention when it crosses a meridian.

The final aspect in which the opposite location differed from the orthogonal locations has to do with the position of the locations relative to the movement of attention. In the present experiment, attention was drawn to a peripheral location with the use of a peripheral cue and then rereoriented to the fixation location with the fixation cue. Thus, moving attention to the opposite location would involve moving attention farther down a path it was already traveling and would not require a change in direction. Moving attention to one of the orthogonal locations would, however, require a $90^{\circ}$ change in direction. It is possible that the faster responses made in the opposite condition occurred because attention has something akin to "momentum" and is more easily allocated along the direction already traveled than along a new direction.

Although the present experiment eliminated the possibility that RTs to targets at the various uncued locations would be the same, the experiment cannot shed any light on why responses in the opposite condition were faster than responses in the orthogonal condition. The following experiments were designed to examine which of the three remaining hypotheses (i.e., spreading inhibition, inhibited hemifield, and attentional momentum) might best account for the pattern of response latencies at the uncued locations.

\section{Experiment 2}

The results from the first experiment, with the RTs in the opposite condition being faster than those in the orthogonal conditions, were consistent with the spreading inhibition (location furthest from cued location the least inhibited), inhibited hemifield (location sharing fewest hemifields with cued location the least inhibited), and attentional momentum (location along the path of attention should be fastest) hypotheses. We designed the present experiment to gain some insight into which of the three hypotheses would provide the best account of the faster RTs for targets appearing at the location opposite the cue.

The method used in Experiment 2 was similar to the method used in Experiment 1 except for one important difference. Unlike the peripheral locations in the first experiment, where all the locations were equally eccentric from the fixation point, the locations in the present experiment were at one of two different eccentricities. Two of the locations were aligned along one diagonal, with each location $7.4^{\circ}$ from the fixation point. The other two locations were aligned along the other diagonal, with each location $4.3^{\circ}$ from the fixation point. Thus, when a cue appeared at one of the near location placeholders, the distance between the cued location and the opposite location was the same as the distance between the cued location and both of the orthogonal locations (i.e., the distance between the cued location and any of the three uncued locations was $8.6^{\circ}$ ). There was also $8.6^{\circ}$ of visual angle between the orthogonal locations when the first cue appeared at one of the distant locations. Therefore, all orthogonal conditions could be collapsed together regardless of where the initial cue was presented.

By holding the distance between the cued and uncued locations constant, it was possible to compare the spreading inhibition hypothesis with the inhibited hemifield and attentional momentum hypotheses. If inhibition tends to spread from the cued location, then uncued locations at equal distances from the cued locations should receive equal amounts of the spreading inhibition. This, in turn, should result in equal RTs to detect the appearance of the targets at the uncued locations.

The inhibited hemifield and attentional momentum hypotheses, however, both predict that responses to targets at the opposite location will be faster than responses at the orthogonal locations. In the case of the inhibited hemifield hypothesis, when the cue appeared in one of the locations near the target, the two orthogonal locations each shared one visual hemifield with the cued location, whereas the opposite location shared no visual hemifields with the cued location. Assuming that inhibition tends to spread within a cued hemifield, targets appearing at the orthogonal locations should be detected more slowly than a target at the opposite location. In the case of the attentional momentum hypothesis, targets appearing at the opposite location should be detected faster because attention has already moved in a path directly toward that location. However, detecting a target at one of the orthogonal locations would require a $90^{\circ}$ change in the direction of the attentional movement.

Overall, our predictions for the present experiment are straightforward. If the spreading inhibition hypothesis best accounts for the faster responses for targets in the location opposite the cued location, then no differences in RT between the opposite and orthogonal conditions should be found in the present experiment when one of the near placeholders is the cued location. However, if responses in the opposite condition are faster than those in the orthogonal condition, then this would negate the spreading inhibition hypothesis and support the inhibited hemifield and attentional momentum hypotheses. 


\section{Method}

Participants. Thirteen undergraduate students from the University of Toronto participated in the study for course credit. Each observer participated in a single session that lasted approximately $30 \mathrm{~min}$.

Apparatus and procedure. The apparatus and procedure were the same as those used in Experiment 1. The only change from the first experiment was that the spatial position of the peripheral target locations was different. The display used in Experiment 2 is shown in Figure 1. Unlike in the first experiment, the peripheral target locations were not equidistant from the fixation point. Instead, two placeholders were aligned on each of two diagonals. Along one diagonal, the placeholders were located $7.4^{\circ}$ from the fixation point. Along the other diagonal, the placeholders were located $4.3^{\circ}$ from the fixation. As in the first experiment, the target could appear at the same location that the cue appeared in (cued condition), at one of the uncued locations to the left or right of the cued location (orthogonal condition), or at the uncued location directly opposite the cued location (opposite condition). However, in this experiment there were two opposite conditions. If the cue appeared in one of the locations that was $7.4^{\circ}$ from the fixation, the opposite location was $14.8^{\circ}$ from the cued location (long opposite condition). If the cue appeared in one of the locations that was $4.3^{\circ}$ from the fixation, the opposite location was $8.6^{\circ}$ from the cued location (short opposite condition). Importantly, the distance between the cued location and the location in the short opposite condition was the same as that between the cued location and either the left or right uncued locations in the orthogonal condition. The remainder of the procedures were the same as those used in Experiment 1.

Design. The experiment consisted of a total of 320 trials per participant. On 64 of those trials, the target appeared in the same location in which the cue had previously appeared (cued condition). On 32 trials, the cue appeared in one of the locations $7.4^{\circ}$ from the fixation point, and the target appeared in the opposite location (long opposite condition). On 32 trials, the cue appeared in one of the locations $4.3^{\circ}$ from the fixation point, and the target appeared in the opposite location (short opposite condition). On 128 trials, the target occurred at the location to the left or right of the cued location (orthogonal condition). There were also 64 catch trials in which no target was presented. The conditions were completely randomized across the experiment, with the cue and target being equally likely to appear at any of the four peripheral locations (and their positions determined completely independently of one another).

\section{Results and Discussion}

The mean RTs from the correct trials appear in Figure 3. The mean RTs were analyzed with a one-way ANOVA using the four conditions (cued, long opposite, short opposite, and orthogonal). Overall, there was a main effect for condition, $F(3,36)=53, p<.00001$, with responses in the cued condition the slowest $(357 \mathrm{~ms})$, responses in the short opposite condition the fastest ( $304 \mathrm{~ms}$ ), and responses in the long opposite and orthogonal conditions falling in between (314 and $322 \mathrm{~ms}$, respectively). As in the first experiment, the finding that the slowest responses occurred in the cued condition confirms that an inhibition-of-return effect was present. However, the most important analysis is the comparison between the two uncued conditions that were equally eccentric from the cued location, the short opposite and orthogonal conditions. A separate $t$ test confirmed that

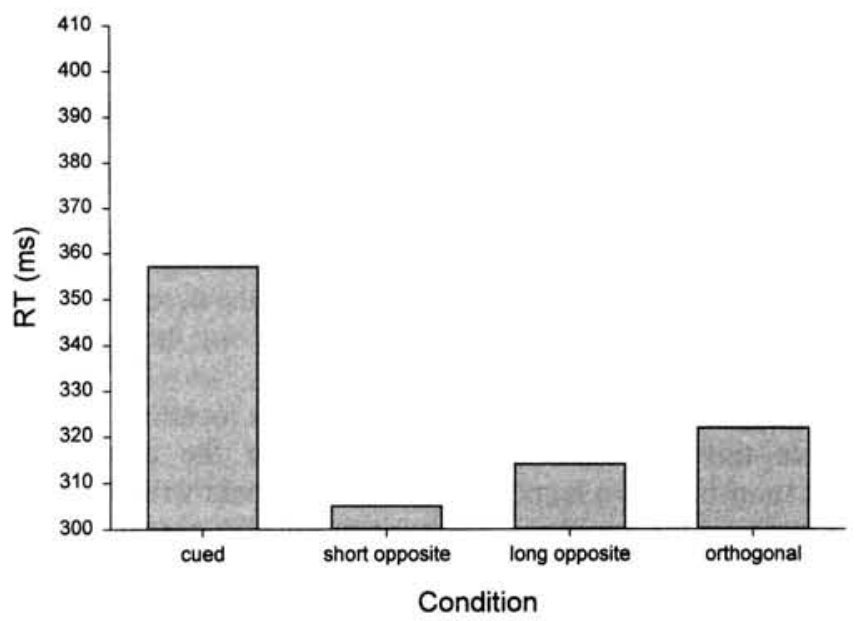

Figure 3. The mean reaction times (RTs) for the correct trials from Experiment 2.

response latencies were faster to targets in the short opposite condition than to targets in the orthogonal condition, $t(12)=$ $4.7, p<.0006$.

The same analysis was conducted on the percentage of error trials, and no main effect was found $(F<1)$. The percentages of error trials in Experiment 2 appear in Table 1.

The findings of the present experiment contradict the spreading inhibition hypothesis. If inhibition simply spread out from a previously attended location, target locations at equal distances away should receive equal inhibition. Despite the fact that the distances between the cued locations and the target locations were equal, the results of the present experiment indicate that responses were faster in the short opposite condition than in the orthogonal conditions. Further evidence against the spreading inhibition hypothesis was provided by the finding that latencies in the short opposite location were faster than latencies in the long opposite condition, $t(12)=2.7, p<.02$. This finding indicates that targets that occurred at a closer eccentricity to the cued location were detected faster than targets that occurred at a farther eccentricity. If inhibition spreads outward from a previously attended location and if the inhibition decays over time and distance, the farther target location should have received less inhibition and this should have resulted in faster responses in the long opposite location. Thus, there is converging evidence that the spreading inhibition hypothesis cannot account for the response latencies to targets at the uncued locations.

It should be noted that there was a necessary eccentricity confound in the present experiment because the various locations (cued, opposite, and orthogonal) were not all equally distant from the fixation location. The effect of this eccentricity difference can be seen in the faster latency responses to the short opposite location than to the long opposite location. However, when eccentricities are averaged across conditions, the opposite conditions still yielded faster RTs ( $309 \mathrm{~ms}$ ) than did the orthogonal $(322 \mathrm{~ms}$ ) or cued (357 ms) locations. Additionally, an advantage for opposite 
locations over orthogonal locations was found in Experiment 1, where the target eccentricity was held constant. Taken together, these findings suggest that the oppositelocation advantage found in the present experiment was not due to an artifact caused by unequal target eccentricities.

Although the present results rule out the spreading inhibition hypothesis, they do not indicate which of the remaining two hypotheses might best account for the data. In the present experiment, the orthogonal locations shared one hemifield with the cued location, whereas the opposite locations did not. Also, the opposite locations were along the path that attention had moved going from the cued location to the fixation point, whereas the orthogonal locations were $90^{\circ}$ off that path. Thus, both the inhibited hemifield and attentional momentum hypotheses would have predicted faster responses for the opposite locations over the orthogonal locations.

\section{Experiment 3}

The results from the previous two experiments indicate that only the inhibited hemifield and attentional momentum hypotheses can account for the findings that response latencies are fastest when targets appear in the uncued location opposite the cued location. We designed Experiment 3 to examine which of these two remaining hypotheses would best account for the response latencies at uncued locations. To do so, in Experiment 3 we used a paradigm unlike that used in the previous experiments.

In Experiment 3, the display consisted of eight placeholder boxes that were at equal distances from the fixation point. Moreover, the distance between the placeholders was also equal, and the placeholders were arranged such that two placeholders appeared in each quadrant. A quadrant consisted of the area overlapped by any two of the four visual hemifields (upper, lower, left, and right hemifields). Following the peripheral cue and the fixation cue, the target could occur at any of the eight potential target locations. Response latencies were expected to be longest when the target occurred at the cued location (the typical inhibition-of-return effect). Importantly, the predictions of the two hypotheses diverge in relation to the seven remaining uncued locations.

The inhibited hemifield hypothesis suggests that inhibition tends to spread within a hemifield and that locations that share more hemifields with the previously attended location will receive more inhibition. Thus, if the cue appeared in the upper left quadrant, the other location in the upper left quadrant should receive the most inhibition (i.e., it shares two hemifields), the locations in the lower left and upper right quadrants should receive less inhibition (i.e., they share one hemifield), and locations in the lower right quadrant should receive little or no inhibition (i.e., they do not share a hemifield with the cued location). A strong version of this hypothesis would predict that the four locations that share one hemifield with the cued location should receive approximately equal inhibition (i.e., should yield approximately equal RTs) and that the two locations that do not share any hemifield with the cued location should not receive any inhibition (and thus yield approximately equal RTs).
Conversely, the attentional momentum hypothesis predicts that the fastest response latencies should be found for targets occurring in the location exactly opposite the cued location because that is the direction in which attention moved from the peripheral cue to the fixation cue. This hypothesis would also predict that locations at equal angles from the direction of attention (from the peripheral cue to the fixation cue) would yield equal response latencies. In other words, the locations immediately adjacent to the cued location should yield equal RTs, as should the locations immediately adjacent to the opposite location and the two locations that are halfway in between the cued and opposite locations.

\section{Method}

Participants. Twenty-five undergraduate students from the University of Toronto participated in the study for course credit. Each student participated in a single session that lasted approximately $40 \mathrm{~min}$.

Apparatus and procedure. The apparatus was the same as that used in the previous experiments. The display used in Experiment 3 is shown in Figure 1 and consisted of eight placeholders located on an imaginary circle around a central fixation point with a radius of $5.5^{\circ}$. Each placeholder was $3.8^{\circ}$ from the adjacent placeholders. The procedure was the same as that used in the previous experiments. In the same manner as in the previous experiments, the target could appear at the same location where the cue appeared (cued condition) or at the uncued location directly opposite the cued location (opposite condition). In addition, the target could appear at any of the other six uncued locations. These locations were designated by how many hemifields they shared with the cued location and by their location relative to the opposite location (how many locations adjacent to the opposite location). Thus, the target could appear in the hemifield, 1-adjacent condition (one location); the 1-hemifield, 1-adjacent condition (one location); the 1-hemifield, two-adjacent condition (two locations); the 1-hemifield, 3-adjacent condition (one location); or the 2-hemifield, 3-adjacent condition (one location). See Figure 4 for details. The remainder of the procedures were the same as those used in Experiments 1 and 2.

Design. The experiment consisted of a total of 360 trials per participant. There were a total of eight conditions, the 7 conditions corresponding to the relationships between the cue and target locations mentioned above and a catch trial condition in which no target was presented. All eight conditions were equally likely during the experiment ( 40 trials per condition), except the 1-hemifield, 2-adjacent condition, which had 40 trials at each of two locations (for a total of 80 trials). The locations of the cue and target were equally likely to occur at any of the eight peripheral locations and were completely independent of one another.

\section{Results and Discussion}

The mean RTs from the correct trials appear in Figure 4. The mean RTs were analyzed with a one-way ANOVA using the seven conditions (i.e., the cued; opposite; 0 -hemifield, 1-adjacent; 1-hemifield, 1-adjacent; 1-hemifield, 2-adjacent; 1-hemifield, 3-adjacent; and 2-hemifield, 3-adjacent conditions). Overall, there was a main effect for condition, $F(6$, $144)=19, p<.00001$, with responses in the cued condition the slowest $(351 \mathrm{~ms})$ and responses in the opposite condition the fastest (322 ms). Although this replicates the findings of 


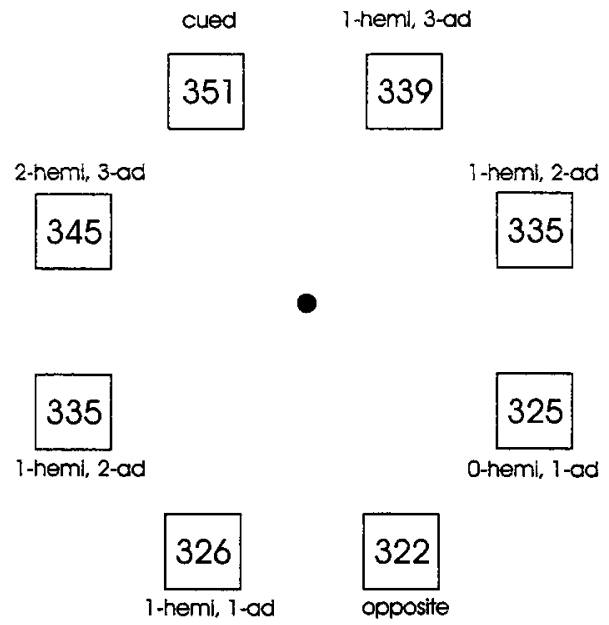

Figure 4. The mean reaction times (RTs) for the correct trials from Experiment 3. This figure shows the RTs for each of the locations collapsed over cue location. For purposes of the figure, the cued location is shown as the top left location, the same as in Figure 1. All RTs are in milliseconds. hemi = hemifield; ad = adjacent.

the previous experiments, the predictions of the inhibited hemifield and attentional momentum hypotheses rest on a series of pairwise comparisons.

The same analysis was conducted on the percentages of trials in which an error occurred, and no main effect for condition was found $(F<1)$. The percentages of error trials appear in Table 1.

The first pairwise comparison examined the latencies to targets at the two locations immediately adjacent to the opposite location. One of these locations shares a hemifield with the cued location (1-hemifield, 1-adjacent condition), whereas the other does not $(0$-hemifield, 1 -adjacent condition). The prediction from the inhibited hemifield hypothesis is that responses in the 1-hemifield, 1-adjacent condition should be slower, whereas the attentional momentum hypothesis predicts that there should be no difference in RT between the two conditions. A paired $t$ test revealed that responses in the 0-hemifield, 1-adjacent condition (326 ms) did not differ from those in the 1-hemifield, 1-adjacent condition ( $325 \mathrm{~ms} ; t<1)$, which supports the attentional momentum hypothesis. Note that responses in the opposite condition were not significantly faster than responses at either of the immediately adjacent conditions, $t \mathrm{~s}(24)<1.5$, $p s>.15$. However, responses in the opposite condition were faster than responses in the other remaining conditions, $t \mathrm{~s}(24)>3.8, p \mathrm{~s}<.0001$. On the one hand, this finding can be accounted for with the attentional momentum hypothesis if the angle of deviation away from the path of attention was not large enough to produce a change in RT. On the other hand, this finding is difficult to reconcile with the inhibited hemifield hypothesis because the opposite location did not share a hemifield with the cued location, unlike one of the immediately adjacent locations.

The second pairwise comparison examined the latencies to the targets at the location farthest away from the opposite location, the 2-hemifield, 3-adjacent condition and the 1-hemifield, 3-adjacent condition. Much like the previous prediction, the inhibited hemifield hypothesis predicts that responses in the 2-hemifield, 3-adjacent condition should be slower, whereas the attentional momentum hypothesis predicts that there should be no difference in RT between the two conditions. A paired $t$ test revealed that response latencies in the 1-hemifield, 3-adjacent condition (339 ms) did not differ from those in the 2-hemifield, 3-adjacent condition (345 ms), $t(24)=2.0, p>.06$, although there was a trend for slower responses in the condition that shared two hemifields with the cued location. This trend remained at a constant level $(p<.06)$ after $10,15,20$, and 25 participants. The two remaining locations that were an equal distance away from the opposite condition (1-hemifield, 2-adjacent) had RTs that were equal ( $335 \mathrm{~ms}$ each; $t<1$ ), but both of the hypotheses would have predicted this result.

Overall, the results from the present experiment provide more support for the attentional momentum hypothesis than for the inhibited hemifield hypothesis. However, this support must be qualified in two ways. First, there was a trend for more inhibition in the 2-hemifield, 3 -adjacent condition than in the 1-hemifield, 3-adjacent condition, although this trend did not reach significance even after the number of participants was more than doubled. Second, the support for the attentional momentum hypothesis comes from null findings, the lack of differences between the two 1-adjacent conditions and the two 3-adjacent conditions. A much stronger test of the attentional momentum hypothesis would be an experiment in which the prediction involved the presence of a difference between conditions, not the lack of a difference. This was provided in Experiment 4.

\section{Experiment 4}

Although the results from Experiment 3 suggest that the attentional momentum hypothesis might provide the best account for the pattern of response latencies at uncued locations, a stronger test of the hypothesis was needed. Experiment 4 was designed to provide a more rigorous test of the attentional momentum hypothesis. Unlike in the previous experiments, the display in the present experiment consisted of only two peripheral target locations, one on each side of the fixation point. The basic procedure of the present experiment followed that of the previous experiments: A cue at one of the peripheral locations was followed by a cue at the fixation location. However, a third cue was presented near the fixation point shortly after the fixation cue but before the target appeared.

The addition of the third cue allowed for some specific predictions from the attentional momentum hypothesis. On some trials, the third cue appeared on the side of the fixation point opposite the peripheral cue (the opposite-side condition). Thus, the presentations of all the cues would appear to be along the same path. For example, a cue at the right peripheral location would be followed by a fixation cue (presumably causing a leftward movement of attention), which in turn would be followed by the third cue to the left 
of the fixation point (continuing the leftward movement of attention). If attention tends to travel along a path where it has already moved, it should be easier to detect targets at the uncued location than at the cued location. Thus, the attentional momentum hypothesis predicts that the typical inhibition-of-return effect should be found on these trials.

On other trials, the third cue appeared on the same side of the fixation point as the peripheral cue (same-side condition). For example, a cue at the right peripheral location would be followed by a fixation cue (presumably causing a leftward movement of attention), which in turn would be followed by the third cue to the right of the fixation point (reversing the path of attention back to the right). This could result in two possible outcomes that are consistent with the attentional momentum hypothesis. One outcome might be that the third cue could reverse the "momentum" of attention and that targets at the cued location would be detected faster than targets at the uncued location. The other outcome would be that the third cue might disrupt the momentum of attention that was directed toward the uncued location such that there would be no disadvantage in detecting targets at either the cued or uncued locations.

If the attentional momentum hypothesis does account for the latencies to targets at uncued locations, a significant interaction should be found between the location of the target (cued or uncued) and the location of the third cue (on the same side as the peripheral cue or on the opposite side of the peripheral cue). Thus, the present experiment provides a stronger test of the attentional momentum hypothesis by allowing for a prediction of a significant difference between conditions, not a null difference between conditions.

\section{Method}

Participants. Twelve undergraduate students from the University of Toronto participated in the study for course credit. Each student participated in a single session that lasted approximately 30 $\min$.

Apparatus and procedure. The apparatus was the same as that used in the previous experiments. The trial sequence used in Experiment 4 is shown in Figure 5. The display consisted of a central fixation point and two horizontally aligned placeholders. After the display was presented for $1,200 \mathrm{~ms}$, a cue was presented at one of the peripheral locations for $200 \mathrm{~ms}$ and then removed. After a 200-ms delay, the fixation cue was presented for $200 \mathrm{~ms}$ and then removed. After another $200 \mathrm{~ms}$, a third cue was also presented for $200 \mathrm{~ms}$. After a final 150-ms delay, a target appeared at one of the peripheral locations. As in the previous experiments, all of the cues consisted of a filled circle, and the target was a filled square.

The location of the placeholders depended on the location of the third cue. The third cue appeared at a position $1^{\circ}$ to either the right or left of the fixation point. To ensure that the peripheral locations were an equal distance from the last attended location, we put the placeholders $5.5^{\circ}$ to the left and right of the location where the third cue appeared on each particular trial. Thus, the fixation point was either $1^{\circ}$ to the left or right of the midpoint between the two peripheral locations.

Unlike in the previous experiments, the participants were instructed to respond to the appearance of the target with a choice manual keypress. If the target appeared in the left location, participants were to press the $z$ key with their left hands. If the

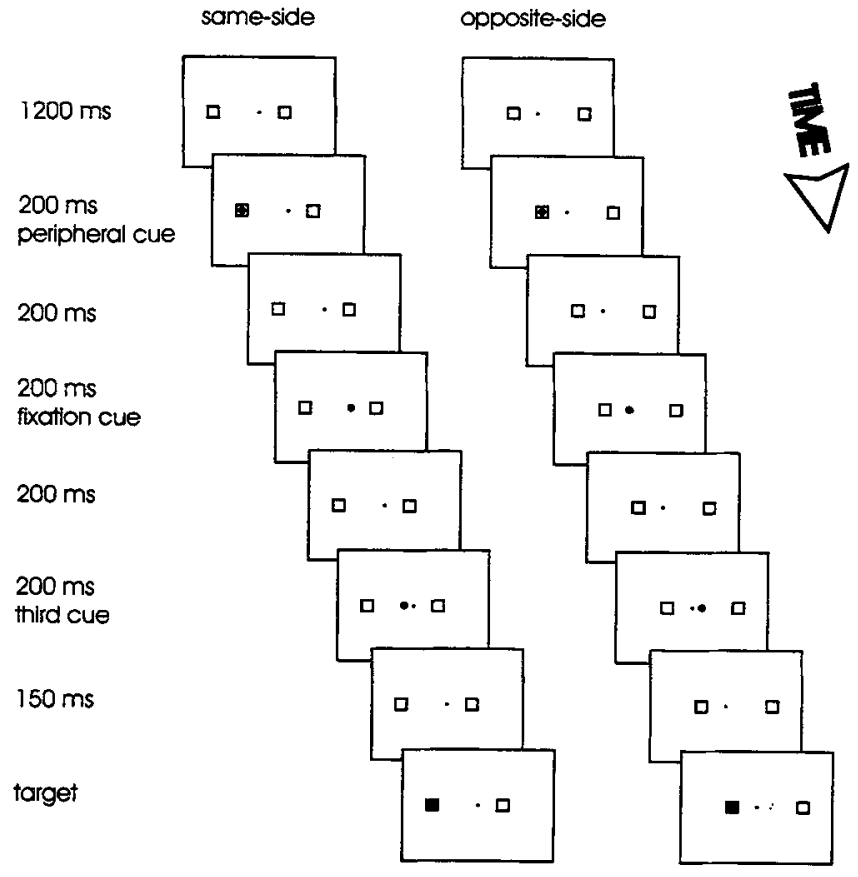

Figure 5. The trial sequence used in Experiment 4. The placeholders and stimuli are not drawn to scale. The peripheral cues and targets were equally likely to occur in either peripheral location, and the third cue was equally likely to appear on the same side as or on a different side from the peripheral cue.

target appeared in the right location, they were to press the forward slash $(I)$ key with their right hands. With the use of choice responses, trials in which participants pressed the wrong key were also counted as errors. Because the responses involved spatial uncertainty, we did not use catch trials in this experiment.

Design. The experiment consisted of a total of 320 trials per participant. There were a total of four conditions because the third cue could occur on the same side as the peripheral cue (same-side condition) or on the opposite side of the peripheral cue (oppositeside condition), and the target could occur at either the cued location (cued condition) or the uncued location (uncued condition). Each combination of these conditions (location of third cue and location of target) was equally likely, resulting in 80 trials per third cue and target combination. The location of the cue was also equally likely to occur at either of the two peripheral locations.

\section{Results and Discussion}

The mean RTs from the correct trials appear in Figure 6. The mean RTs were analyzed with a 2 (third cue: same side or opposite side) $\times 2$ (target: cued or uncued) ANOVA. No main effects were found for the location of the third cue $(F<1)$ or for the location of the target, $F(1,11)<1.6, p>$ .24. Importantly, the interaction was significant, $F(1,11)=$ $8.0, p<.02$, because RTs were faster if the target occurred at the uncued location in the opposite-side condition and at the cued location in the same-side conditions. To further examine this interaction, we conducted pairwise $t$ tests. These analyses revealed the typical inhibition-of-return effect in the opposite-side condition, with slower responses to targets 


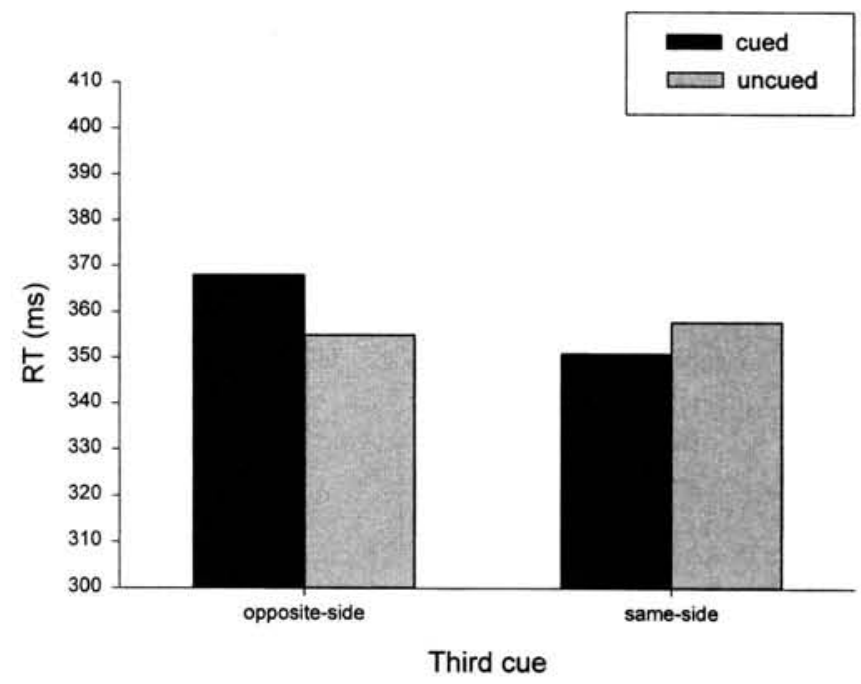

Figure 6. The mean reaction times (RTs) for the correct trials from Experiment 4.

at the cued ( $368 \mathrm{~ms})$ than at the uncued $(355 \mathrm{~ms})$ locations, $t(11)=3.9, p<.003$. However, the difference in the same-side condition between cued $(351 \mathrm{~ms})$ and uncued (358 ms) target locations was not significant, $t(11)<1.5$, $p>.21$.

The same analysis was conducted on the percentages of trials in which an error occurred, and no main effect was found for third cue or target $(F \mathrm{~S}<1)$. The percentages of error trials appear in Table 1.

The finding of inhibition of return in the opposite-side condition and not in the same-side condition is consistent with the predictions of the attentional momentum hypothesis. The attentional momentum hypothesis suggests that the time to respond to the appearance of a target depends on the relationship between the target location and the direction of travel of attention. In other words, targets appearing at locations along the path where attention has already moved will be responded to faster than targets that occur at locations that require a change in the direction of attention. In the present experiment, attention traveled from the peripheral cue to the fixation cue to the third cue. Thus, in the opposite-side condition, the uncued location occurred along the path of attention, whereas the cued location was in the opposite direction. Consequently, faster response latencies were found at the uncued location. In the same-side condition, however, the third cue appeared in the direction opposite to the direction that attention had moved in from the peripheral cue to the fixation cue. According to the attentional momentum hypothesis, this should either have reversed the direction of travel of attention or at least disrupted the path of travel. The findings suggest that the latter effect occurred, because the typical inhibition-ofreturn effect was not found; instead, targets at the cued and uncued locations were not responded to with different detection latencies.

Note that the inhibited hemifield hypothesis remained a possibility in the present experiment because of the lack of strong conflicting evidence found in Experiment 3. However, this view cannot account for the pattern of results found in Experiment 4 . This is because only two peripheral locations were used in Experiment 4, one in each of the left and right hemifields. Thus, the uncued location did not share a hemifield with the cued location on any of the trials. The inhibited hemifield hypothesis would have predicted that responses would have been faster for targets at the uncued location, regardless of the location of the third cue, because the spread of inhibition would have been limited to the cued hemifield. The finding of inhibition of return in the oppositeside condition but not the same-side condition is clearly inconsistent with the inhibited hemifield hypothesis.

Although the results of the fourth experiment argue against the inhibited hemifield hypothesis, it is possible that the results were due not only to attentional momentum but also to spreading inhibition. It may have been that inhibition spread from the fixation location because of the delay between the cuing of the fixation location and presentation of the target (i.e., after the third cue, the fixation location became a previously attended location). Because the cued location was closer to the fixation cue than to the uncued location in the opposite-side condition, any spread of inhibition from the fixation location would have affected the cued location more than the uncued location. Conversely, in the same-side condition, any inhibition from the fixation cue would have affected the uncued location more than the cued location. To separate the effects of spreading inhibition from attentional momentum, we conducted a fifth experiment.

\section{Experiment 5}

Experiment 5 had two purposes. The first purpose was to determine whether the interaction found in Experiment 4 was due to attentional momentum or spreading inhibition. We did this by making two important changes in the experimental method from Experiment 4. One change was that the fixation cue appeared after the "third cue" (to keep the terms constant across experiments, we use the term third cue despite the fact that in Experiment 5 the order of the cue presentations was peripheral cue, third cue, fixation cue, and then the target). Thus, in Experiment 5, the attentional momentum hypothesis would predict inhibition of return in the same-side condition and not in the opposite-side condition (the reverse of the predictions from Experiment 4). The other change was that the target locations were located equidistant from the fixation point.

The other purpose of Experiment 5 was to eliminate the possibility that the previous effects were due to overt movements of attention (i.e., eye movements). To do so, we monitored the participants' eyes in the present experiment; trials in which their gazes moved away from the fixation point were considered errors, and the data from those trials were not analyzed.

\section{Method}

Participants. Thirteen undergraduate students from the University of Toronto participated in the study for course credit. Each 
student participated in a single session that lasted approximately $60 \mathrm{~min}$.

Apparatus and procedure. The apparatus was the same as that used in the previous experiments with the addition of a scleralreflectance eye movement monitor (Model 210, Applied Science Laboratories, Bedford, MA). The trial sequence was the same as that used in Experiment 4 except that the presentations of the fixation cue and the third cue were switched such that the third cue was presented before the fixation cue. This is shown in Figure 5. The display was also the same as that used in Experiment 4 except that the two peripheral target locations were centered on the fixation location and not on the location of the third cue (i.e., $5.5^{\circ}$ to the left and right of the fixation point).

Eye movement monitoring. The eye movement monitor was calibrated at the beginning of each session, and the calibration was checked at the beginning of each trial. Participants' eye positions were monitored at four different times during the trial (i.e., after the onset of the peripheral cue; after the onset of the third cue; after the onset of the fixation cue; and after the onset of the target). If the participant's eyes were more than $1.0^{\circ}$ from the fixation location at any of these monitored times during a trial, an error message was provided to the participant and the trial was eliminated from the analysis.

Design. The experiment consisted of a total of 320 trials per participant. There were a total of four conditions because the third cue could occur on the same side as the peripheral cue (same-side condition) or on the opposite side of the peripheral cue (oppositeside condition), and the target could occur at either the cued location (cued condition) or the uncued location (uncued condition). Each combination of these conditions (location of third cue and location of target) was equally likely, resulting in 80 trials per third cue and target combination. The location of the cue was also equally likely to occur at either of the two peripheral locations.

\section{Results and Discussion}

The mean RTs from the correct trials appear in Figure 7. The mean RTs were analyzed with a 2 (third cue: same side or opposite side) $\times 2$ (target: cued or uncued) ANOVA. No main effect was found for the location of the third cue

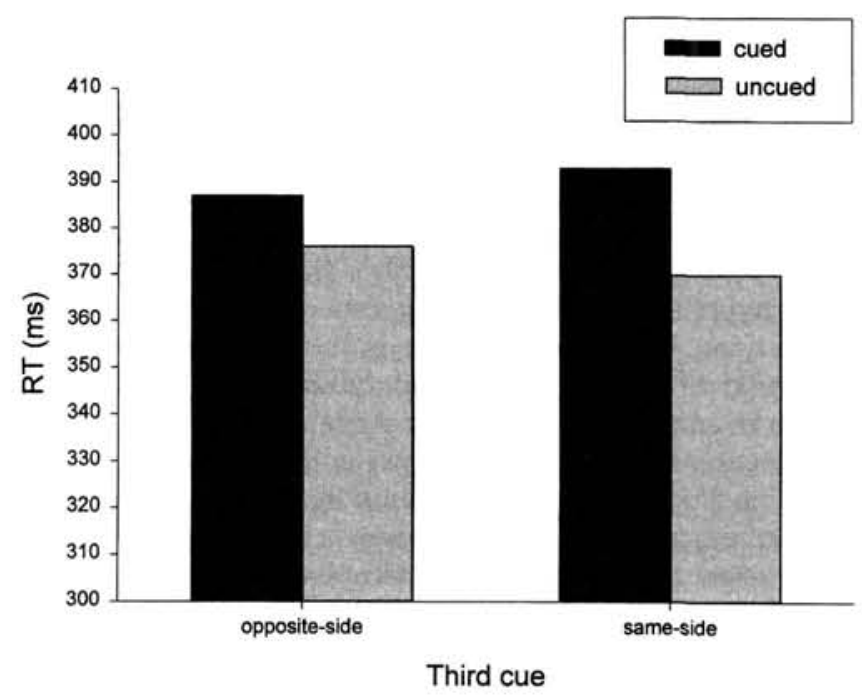

Figure 7. The mean reaction times (RTs) for the correct trials from Experiment 5.
$(F<1)$, but cued trials yielded longer RTs than did uncued trials, $F(1,12)=11.9, p>.005$. Importantly, the interaction was significant, $F(1,12)=5.6, p<.04$, because more inhibition was found in the same-side condition than in the opposite-side condition. To further examine this interaction, we conducted pairwise $t$ tests. These analyses revealed the typical inhibition-of-return effect in the same-side condition, with slower responses to targets at the cued $(393 \mathrm{~ms})$ than at the uncued $(370 \mathrm{~ms})$ locations, $t(12)=4.6, p<.001$. Although the difference in the opposite-side condition between cued $(387 \mathrm{~ms})$ and uncued $(375 \mathrm{~ms})$ target locations was not significant, $t(12)<2.0, p\rangle .10$, there was a trend for inhibition in this condition.

The same analysis was conducted on the percentages of trials in which an error occurred, and no main effect was found for third cue or target, $F \mathrm{~s}(1,12)<1.7, p s>.21$. The percentages of error trials appear in Table 1 .

Overall, the results from the fifth experiment are consistent with the predictions from the attentional momentum hypothesis. With the possibility of spreading inhibition controlled for, inhibition of return was found only in the same-side condition. Thus, the results from the present experiment replicate the results from Experiment 4 while controlling both for spreading activation and for the overt orienting of attention with eye movements.

Note, however, that a strong version of the attentional momentum explanation would predict faster response latencies at the cued location than at the uncued location. This was not found in the present experiment because, although not statistically different, RTs to the cued location were longer than RTs to the uncued location. There is, however, some evidence to suggest that inhibition of return may not be strictly an attentional phenomenon but may also be due to inhibition in the motor system (e.g., Klein \& Taylor, 1994). The possibility that inhibition of return consists of a motor inhibition component and an attentional inhibition component is discussed in the General Discussion section.

\section{General Discussion}

We designed the present study to gain some insight into which of four attentional hypotheses (i.e., inhibited location, spreading inhibition, inhibited hemifields, and attentional momentum) would best account for the pattern of detection latencies at previously attended and unattended peripheral locations. Experiment 1 showed that the latency to detect a target differed among the three uncued locations, a result inconsistent with the inhibited location hypothesis. Experiment 2 showed that detection latencies for targets at the orthogonal uncued locations were slower than those at the opposite location despite the locations' being equally eccentric from the cued location. This result was inconsistent with the spreading inhibition hypothesis but consistent with both the inhibited hemifield and attentional momentum hypotheses. In the final three experiments we sought to determine which of the two remaining hypotheses would best predict the RTs at uncued locations. Experiment 3 showed that latencies to targets at equal angles off the path of attention had approximately equal RTs, although there was a trend for 
slower latencies at locations that shared two hemifields with the cued location. In a stronger test of the attentional momentum hypothesis, Experiment 4 showed that inhibition of return occurred when the direction of attention was toward the uncued location and that inhibition of return did not occur when the direction of attention was reversed toward the cued location. Finally, Experiment 5 replicated the results of Experiment 4 while controlling for the possibility of spreading inhibition and the overt orienting of attention with eye movements. Overall, the results of the present experiments suggest that the attentional momentum hypothesis provides the best account of the pattern of detection latencies for targets presented at cued and uncued locations.

\section{Implications for Inhibition of Return}

The finding of support for the attentional momentum hypothesis suggests that although attention may be inhibited from returning to a previously attended location, attention is also facilitated in moving toward certain unattended locations. The traditional explanation for the difference in RT between the detection of targets presented at cued and uncued locations is that attention is inhibited from returning to the cued location. It is implicitly assumed in this explanation that the time to detect targets at the uncued location is not under any particular attentional influence and thus represents a "typical" or "baseline" time of target detection. Thus, the RT difference between targets presented at cued and uncued locations is thought to be solely due to inhibition at the cued location. The present results suggest that this characterization of what occurs at cued and uncued locations may not be correct. Rather than thinking of inhibition of return as solely reflecting an inhibitory process, a better way of thinking about it might be as a process involved in reorienting the direction of attention. Targets occurring in the same direction as the previous movement of attention (in other words, along the path that attention moved in traveling from the peripheral cue to the fixation cue) are detected faster than targets that are $180^{\circ}$ opposite the direction of the previous movement of attention. In other words, the typical inhibition-of-return effect may be the time needed to change the direction of attention $180^{\circ}$.

Note that the notion that the mechanism underlying inhibition of return may be the time needed to reorient the direction of the path of attention (what we have called attentional momentum) is not inconsistent with the traditional notion that attention is inhibited from returning to previously attended locations. Rather, attentional momentum might best be considered as a more complete explanation of the inhibition-of-return effect, an explanation that recognizes that there are attentional effects at both cued and uncued locations.

The attentional momentum explanation is, obviously, consistent with the previous notion that inhibition of return is an attentional phenomenon. Thus, research that has indicated that factors that affect the attentional system also affect inhibition of return (e.g., Reuter-Lorenz et al., 1996; Tassinari \& Berlucchi, 1993) is consistent with the notion of attentional momentum. Moreover, one prediction arising from the attentional momentum hypothesis is that slower responses to targets will result when the target occurs at a location that requires a change in the direction of attention, regardless of the type of response (simple manual keypress, choice manual keypress, eye movement). As mentioned earlier, inhibition of return has been found for all of these types of responses (e.g., Maylor, 1985; Vaughan, 1984). This prediction also applies to what the response is based on, the detection of the appearance of a target or the discrimination of a specific target stimulus. No matter what the response is based on (e.g., appearance of the target, spatial location of the target, identity of the target), the attentional momentum hypothesis predicts that responses will be slower to targets that require a change in the direction of attention. Thus, the growing evidence that inhibition of return occurs in discrimination tasks (e.g., Chasteen \& Pratt, 1999; Lupiáñez et al., 1997; Pratt, 1995; Pratt \& Abrams, 1999; Pratt et al., 1997) is consistent with the notion of attentional momentum.

The attentional momentum hypothesis may also account for one of the more particular findings recently reported, the lack of inhibition in the double-cued condition in a study by Pratt and Abrams (1995). Pratt and Abrams did not find inhibition of return in trials in which a peripheral cue was followed by two consecutive cues at the fixation location and then a target at either the cued or uncued location. It may have been that the double fixation cue disrupted the path of attention in the same way as the third cue did in the same-side condition of our Experiment 4. The attentional momentum hypothesis would predict that because of this disruption of the path of attention, there would be no advantage to orienting attention to the uncued location and no disadvantage to orienting it to the uncued location. Thus, the results from the double-cued trials of Pratt and Abrams are consistent with the notion of attentional momentum.

Although the attentional momentum hypothesis may be considered a more comprehensive version of the traditional attentional explanation for inhibition of return, it does call into question the notion that inhibition of return reflects an attentional mechanism that improves the efficiency of attentional searches. Posner and Cohen (1984) were the first to suggest that inhibition of return is an adaptive mechanism that biases attention toward novel locations and away from previously attended locations. This notion was later expanded on by several researchers, including Tipper et al. (1991, 1994, 1996) and Pratt (1995). The attentional momentum hypothesis, however, suggests that the mechanism underlying inhibition of return is the reorientation of the direction of attention. Thus, inhibition of return is not so much an adaptive mechanism in attentional search but rather a reflection of the fact that it is easier to orient attention to a location along the path of attention than to locations off the path of attention. Because attention is biased to move along the current path of attention, orienting attention to various locations results in a bias against returning attention to previously attended locations and a bias toward novel locations. Note that unlike the traditional attentional explanation for inhibition of return (that attention is inhibited from returning to previously attended locations), the attentional 
momentum hypothesis suggests that attention will be biased to move to some unattended locations (i.e., locations along the path of attention) faster than to other unattended locations (i.e., locations off the path of attention). In other words, inhibition of return is the difference between orienting attention quickly to some locations and more slowly to other locations.

\section{Attention and Eye Movements}

The reason that targets are detected faster at locations along the path of attention may be due to the manner with which attention is oriented from location to location. There is considerable evidence to suggest that the visual attentional system and the oculomotor system are intimately connected (e.g., Deubel \& Schneider, 1996; Shepherd, Findlay, \& Hockey, 1986). The connections between these two systems have led to the development of the premotor theory of attention by Rizzolatti et al. (1987). Premotor theory suggests that common mechanisms control the orienting of attentional movements and eye movements. In fact, movements of attention are simply unexecuted eye movements in premotor theory because the same mechanisms underlie each type of movement. Importantly, Rizzolati et al. (1987) noted that "in the case of ocular movements a change in direction ... is likely to cause a greater delay than a change in distance" (p. 38) and that similar differences between changing direction and extent should occur for the orienting of attention to various locations. The work of Rafal et al. (1989), indicating that oculomotor activation is a critical component in inhibition of return, provides for a possible link between the attentional momentum hypothesis and the premotor theory of attention. Although premotor theory does appear to be a plausible candidate for why attention might seem to have its own momentum, there is no direct evidence linking inhibition of return to premotor theory at the present time. Indeed, premotor theory has not been proved beyond a shadow of doubt, because Klein (1980) and Klein and Pontefract (1994) failed to find support for the theory. It may be that attentional momentum reflects a more limited connection between the attentional and oculomotor systems, perhaps a connection that involves only reflexive orienting responses to peripheral stimuli or one that requires oculomotor activation. In addition, the present results also suggest that cuing effects in general (i.e., facilitation with short delays between cues and targets, inhibition with longer delays) may be due to attentional momentum. Future research examining the attentional momentum hypothesis will, no doubt, provide some information about the nature of the relationship between the oculomotor system, the orienting of attention, and inhibition of return.

\section{Inhibition of Return for Objects}

Any explanation for inhibition of return must be able to account for the considerable evidence that inhibition of return may be object based. The results of several studies (e.g., Abrams \& Dobkin, 1994; Tipper et al., 1991, 1994) have shown that inhibition remains with a cued object even after the object has moved to a location that was never cued. Consider a typical sequence of events in an object-based inhibition-of-return experiment. An object is cued and then moves slowly to some new location. Presumably, the movement of the object captures attention and the attentional system constantly updates the changing location of the cued object. At some point in the trial, the cued object stops moving and the fixation cue is presented. It is at this point in the trial that attention moves from the cued object toward the fixation location and produces the attentional momentum. Thus, in typical object-based inhibition-of-return tasks, the momentum of attention would be away from the final location of the cued object and toward the directly opposite location.

\section{Inhibition of Return at Multiple Locations}

Although the attentional momentum hypothesis provides a parsimonious explanation for the inhibition-of-return effect and can account for a considerable amount of the previous research findings, there are some results regarding inhibition of return that are not easily accounted for within this hypothesis. For example, several studies have shown that more than one cued location may yield increased detection latencies to targets. This effect has been found with multiple simultaneously cued locations (Wright \& Richard, 1996) and with multiple sequentially cued locations (Abrams \& Pratt, 1996; Danziger et al., 1998; Tipper et al., 1996). It is possible that the attentional momentum hypothesis can account for some of the results found in the experiments with multiple sequential cues because the RTs from the various locations were not examined relative to the path of attention from the last cued peripheral location to the fixation point. In fact, the finding that inhibition to multiple sequentially cued locations occurs only when the multiple locations may be grouped into a single inhibited area (Abrams \& Pratt, 1996) may fit into the attentional momentum hypothesis. Assuming that multiple cued locations are grouped into an inhibited area, it may be that the path of attention extends from a previously attended area. It may be possible to determine the veracity of this suggestion by examining the RTs to the various cued (and uncued) locations individually relative to the path of attention from the last cued location to the fixation point.

However, recent evidence by Danziger et al. (1998) has shown that inhibition of return may be found at multiple sequentially cued locations that may not be grouped into a single inhibited region. In addition, Wright and Richard (1996) found slower detection latencies at one, two, three, and four simultaneously cued locations of eight possible locations. Because the multiple cues appeared at spatially distinct locations in these experiments, it is difficult (if not impossible) to determine the path of attention. It is, of course, possible that multiple paths of attention existed in these studies. This would necessarily involve allocating attention to more than one location at the same time. Although there is some evidence that it is possible to attend 
to two spatially distinct locations (e.g., Kramer \& Hahn, 1996), there is no evidence to suggest that attention may be allocated to four separate locations.

However, there is some evidence that inhibition of return may not represent an entirely attentional phenomenon. Klein and Taylor (1994) suggested that inhibition of return was best characterized as a bias against responding to an inhibited location. A location would become inhibited when a motor program (specifically an oculomotor program) was created for an overt movement toward a cued location, but this program was not initiated and subsequently canceled. Thus, Klein and Taylor suggested that inhibition will occur only for detection responses and discrimination responses based on the spatial location of the target. There is, however, growing evidence that inhibition may be found for responses based solely on the identity of a target, indicating that inhibited spatially localized motor responses cannot provide a complete account for the inhibition found at cued locations (e.g., Chasteen \& Pratt, 1999; Lupiáñez et al., 1997; Pratt et al., 1997).

There is also research that suggests that inhibition of return consists of both an attentional component and a motor component. Abrams and Dobkin (1994) compared keypress responses and eye movement responses to exogenous peripheral targets and endogenous central targets. They found evidence for inhibition strictly within the oculomotor system, because eye movement latencies were increased when a central arrow indicated that an eye movement was to be made to a cued location (despite the fact that a peripheral target was never presented; see also Abrams \& Pratt, 1998). More recently, Kingstone and Pratt (in press) found that in choice keypress tasks involving an identity discrimination, more inhibition was found when eye movements were made to the target location than when the eyes remained fixated. Kingstone and Pratt interpreted this finding as evidence for the presence of an attentional and a motor component for inhibition of return (i.e., the motor component being the difference in the amount of inhibition between the eyesfixated and the eyes-move tasks).

The notion that inhibition of return consists of both an attentional component (represented by attentional momentum) and a motor component (represented by inhibited locations) can provide a thorough account of the phenomenon. After every peripheral cue, a reflexive eye movement is planned but is not initiated. This results in a relatively small amount of inhibition at every cued location. After the removal of attention from the last cued location to the center fixation location, the momentum of attention creates a bias for attention to move in a specific direction (i.e., opposite that of the last cued location). Therefore, the magnitude of the inhibitory effect is a combination of the relatively constant amount of motor inhibition (each motor program canceled would presumably produce similar amounts of inhibition) and the relatively variable attentional inhibition (the amount of this inhibition would depend on the location of any given target relative to the attentional momentum). In fact, some evidence for such a notion can be found in the present study. Recall that in Experiment 5, a trend toward an inhibitory effect was found in the away condition, a result not predicted by the attentional momentum hypothesis. It may be that the small amount of inhibition observed in that condition represented the motor inhibition associated with cuing a peripheral location.

\section{Conclusions}

In summary, in the present research we examined the pattern of detection latencies that occurred at both cued and uncued locations in variations of the standard inhibition-ofreturn paradigm. Of the possible attentional explanations, the attentional momentum hypothesis provided the best account for the findings from the present experiments. This hypothesis suggests that the time to detect a target at any given location is determined by the relationship between the given location and the path of attention. The larger the change in the direction of attention, the longer the latency to detect the target. Thus, inhibition of return may be not simply the inhibition of returning attention to a previously attended location but rather the difference between orienting attention to locations on and off the path of attention. Overall, we suggest that a two-component model of inhibition of return, consisting of attentional momentum and motor inhibition, provides a thorough account for the inhibitory effect that follows a peripheral cue.

\section{References}

Abrams, R. A., \& Dobkin, R. S. (1994). Inhibition of return: Effects of attentional cueing on eye movement latencies. Journal of Experimental Psychology: Human Perception and Performance, $20,467-477$.

Abrams, R. A., \& Pratt, J. (1996). Spatially-diffuse inhibition affects multiple locations: A reply to Tipper, Weaver, and Watson. Journal of Experimental Psychology: Human Perception and Performance, 22, 1294-1298.

Abrams, R. A., \& Pratt, J. (1998). Retinal coding of inhibited eye movements to recently attended locations. Manuscript submitted for publication.

Berlucchi, G., Tassinari, G., Marzi, C. A., \& Di Stefano, M. (1989). Spatial distribution of the inhibitory effect of peripheral noninformative cues on simple reaction time to non-fixated visual targets. Neuropsychologia, 27, 201-221.

Chasteen, A. L., \& Pratt, J. (1999). Inhibition of return in a lexical decision task. Psychological Science, 10, 41-46.

Corbetta, M., Miezin, F. M., Shulman, G. L., \& Petersen, S. E. (1993). A PET study of visuospatial attention. Journal of Neuroscience, 13, 1202-1226.

Danziger, S., Kingstone, A., \& Snyder, J. J. (1998). Inhibition of return to successively stimulated locations in a sequential visual search task. Journal of Experimental Psychology: Human Perception and Performance, 24, 1467-1475.

Deubel, H., \& Schneider, W. X. (1996). Saccade target selection and object recognition: Evidence for a common attentional mechanism. Vision Research, 36, 985-996.

Freyed, J. J., \& Finke, R. A. (1984). Representational momentum. Journal of Experimental Psychology: Learning, Memory, and Cognition, 10, 126-132.

Freyed, J. J., \& Finke, R. A. (1985). A velocity effect for representational momentum. Bulletin of the Psychonomic Society, 23, 443-446. 
Gibson, B. S., \& Egeth, H. (1994). Inhibition of return to object-based and environment-based locations. Perception \& Psychophysics, 55, 323-339.

Hawkins, H. L., Shafto, M. G., \& Richardson, K. (1988). Effects of target luminance and cue validity on the latency of visual detection. Perception \& Psychophysics, 44, 484-492.

Hubbard, T. L. (1995). Environmental invariants in the representation of motion: Implied dynamics, gravity, friction, and centripetal force. Psychonomic Bulletin \& Review, 2, 322-338.

Hughes, H. C., \& Zimba, L. D. (1985). Spatial maps of directed visual attention. Journal of Experimental Psychology: Human Perception and Performance, $11,409-430$.

Hughes, H. C., \& Zimba, L. D. (1987). Natural boundaries for the spatial spread of directed visual attention. Neuropsychologia, 25, 5-18.

Kingstone, A., \& Pratt, J. (in press). Inhibition of return is composed of attentional and motor processes. Perception \& Psychophysics.

Klein, R. (1980). Does oculomotor readiness mediate cognitive control of visual attention? In R. Nickerson (Ed.), Attention and performance VIII (pp. 259-276). Hillsdale, NJ: Erlbaum.

Klein, R., \& Pontefract, A. (1994). Does oculomotor readiness mediate cognitive control of visual attention? Revisited! In C. Umilta \& M. Moscovitch (Eds.), Attention and performance $X V$ (pp. 333-350). Cambridge, MA: MIT Press.

Klein, R., \& Taylor, T. L. (1994). Categories of cognitive inhibition with reference to attention. In D. Dagenbach \& T. Carr (Eds.), Inhibitory processes in attention, memory, and language (pp. 113-150). New York: Academic Press.

Kramer, A. F., \& Hahn, S. (1996). Splitting the beam: Distribution of attention over noncontiguous regions of the visual field. Psychological Science, 6, 381-386.

Kwak, H., Dagenbach, D., \& Egeth, H. (1991). Further evidence for a time-independent shift of the focus of attention. Perception \& Psychophysics, 49, 473-480.

LaBerge, D., \& Brown, V. (1986). Variations in size of the visual field in which targets are presented: An attentional range effect. Perception \& Psychophysics, 40, 188-200.

Lupiáñez, J., Milán, E. G., Tornay, F. J., Madrid, E., \& Tudela, P. (1997). Does IOR occur in discrimination tasks? Yes, it does, but later. Perception \& Psychophysics, 59, 1241-1254.

Maylor, E. (1985). Facilitatory and inhibitory components of orienting in visual space. In M. I. Posner \& B. B. Marin (Eds.), Attention and performance XI (pp. 189-204). Hillsdale, NJ: Erlbaum.

Maylor, E., \& Hockey, R. (1985). Inhibitory component of externally controlled covert orienting in visual space. Journal of Experimental Psychology: Human Perception and Performance, $11,777-787$.

Müller, H. J., \& von Mühlenen, A. (1996). Attentional tracking and inhibition of return in dynamic displays. Perception \& Psychophysics, 58, 224-249.

O'Donnell, C. D., \& Pratt, J. (1996). Inhibition of return along the path of attention. Canadian Journal of Experimental Psychology, 50, 386-392.

Posner, M. I. (1980). Orienting of attention. Quarterly Journal of Experimental Psychology, 32, 3-25.

Posner, M. I., \& Cohen, Y. (1984). Components of visual orienting. In H. Bouma \& D. G. Bouwhuis (Eds.), Attention and performance $X$ (pp. 531-556). Hillsdale, NJ: Erlbaum.

Pratt, J. (1995). Inhibition of return in a discrimination task. Psychonomic Bulletin \& Review, 2, 117-120.

Pratt, J., \& Abrams, R. A. (1994). Action-centered inhibition: Effects of distractors on movement planning and execution. Human Movement Science, 13, 245-254.
Pratt, J., \& Abrams, R. A. (1995). Inhibition of return to successively cued spatial locations. Journal of Experimental Psychology: Human Perception and Performance, 21, 1343-1353.

Pratt, J., \& Abrams, R. A. (1999). Inhibition of return in discrimination tasks. Journal of Experimental Psychology: Human Perception and Performance, 25, 229-242.

Pratt, J., Kingstone, A., \& Khoe, W. (1997). Inhibition of return in location and identity based choice decision tasks. Perception \& Psychophysics, 59, 964-971.

Rafal, R. D., Calabresi, P. A., Brennan, C. W., \& Sciolto, T, K. (1989). Saccade preparation inhibits reorienting to recently attended locations. Journal of Experimental Psychology: Human Perception and Performance, 15, 673-685.

Reuter-Lorenz, P. A., Jha, A. P., \& Rosenquist, J. M. (1996). What is inhibited in inhibition of return? Journal of Experimental Psychology: Human Perception and Performance, 22, 367-378.

Rizzolatti, G., Riggio, L., Dascola, I., \& Umilta, C. (1987). Reorienting attention across the horizontal and vertical meridians: Evidence in favor of a premotor theory of attention. Neuropsychologia, 25, 31-40.

Sagi D., \& Julesz, B. (1985). Fast noninertial shifts of attention. Spatial Vision, 2, 141-149.

Shepherd, M., Findlay, J. M., \& Hockey, R. J. (1986). The relationship between eye movements and spatial attention. Quarterly Journal of Experimental Psychology: Human Experimental Psychology, 38A, 475-491.

Shulman, G. L., Remington, R. W., \& McLean, J. P. (1979). Moving attention through visual space. Journal of Experimental Psychology: Human Perception and Performance, 5, 522-526.

Tanaka, Y., \& Shimojo, S. H. (1996). Vision, attention, and action: Inhibition and facilitation in sensory-motor links revealed by the reaction time and the line motion. Vision Research, 36, 21252140.

Tassinari, G., Aglioti, S., Chelazzi, L., Marzi, C. A., \& Berlucchi, G. (1987). Distribution in the visual field of the costs of voluntarily allocated attention and of the inhibitory after-effects of covert orienting. Neuropsychologia, 25, 55-72.

Tassinari, G., \& Berlucchi, G. (1993). Sensory and attentional components of slowing of manual reaction time to non-fixated visual targets by ipsilateral primes. Vision Research, 33, 15251534.

Tassinari, G., \& Campara, D. (1996). Consequences of covert orienting to noninformative stimuli of different modalities: A unitary mechanism? Neuropsychologia, 34, 235-245.

Terry, K. M., Valdes, L. A., \& Neill, T. (1994). Does "inhibition of return" occur in discrimination tasks? Perception \& Psychophysics, 55, 323-339.

Tipper, S. P., Driver, J., \& Weaver, B. (1991). Object-centered inhibition of return of visual attention. Quarterly Journal of Experimental Psychology: Human Experimental Psychology, 43A, 289-298.

Tipper, S. P., Lortie, C., \& Baylis, G. C. (1992). Selective reaching: Evidence for action-centered attention. Journal of Experimental Psychology: Human Perception and Performance, 18, 891-905.

Tipper, S. P., Weaver, B., Jerreat, L. M., \& Burak, A. L. (1994). Object- and environment-based inhibition of retum of visual attention. Joumal of Experimental Psychology: Human Perception and Performance, 20, 478-499.

Tipper, S. P., Weaver, B., \& Watson, F. L. (1996). Inhibition of return to successively cued spatial locations: Commentary on Pratt and Abrams (1995). Journal of Experimental Psychology: Human Perception and Performance, 22, 1289-1293

Vaughan, J. (1984). Saccades directed at previously attended locations in space. In A. G. Gale \& F. Johnson (Eds.), Theoreti- 
cal and applied aspects of eye movement research (pp. 143150). Amsterdam: North-Holland.

Wright, R. D., \& Richard, C. M. (1996). Inhibition of return at multiple locations in visual space. Canadian Journal of Experimental Psychology, 50, 324-327.

Yantis, S., \& Jonides, J. (1990). Abrupt visual onsets and selective attention: Voluntary versus automatic allocation. Journal of
Experimental Psychology: Human Perception and Performance, $16,121-134$.

Received July 28, 1997

Revision received January 22, 1998

Accepted May 4, 1998

\section{American Psychological Association SUBSCRIPTION ClaIMS INFORMATION}

Today's Date:

We provide this form to assist members, institutions, and nonmember individuals with any subscription problems. With the appropriate information we can begin aresolution. If you use the services of an agent, please do NOT duplicate claims through them and directly to us. PLEASE PRINT CLEARLY AND IN INK IF POSSIBLE.

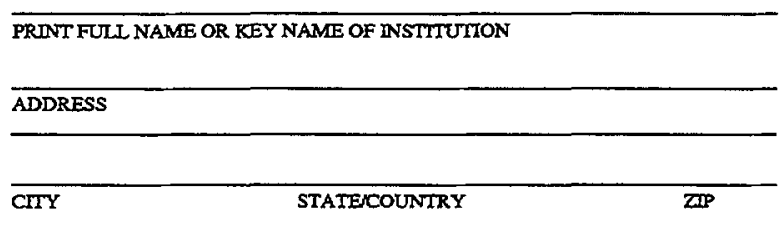

YOUR NAME AND PEONE NUMBER

TILE

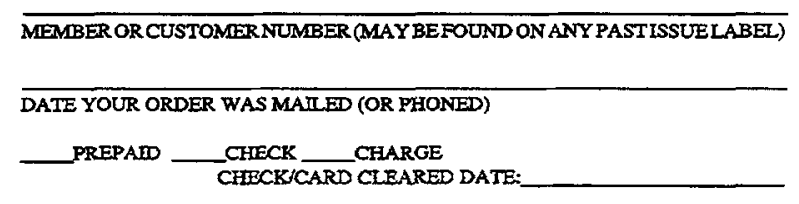

(af possible, send a copy, front and back, of your cancelled chock to belp us in our research of your claim.)

VOLUME OR YEAR
ISSUES: _ MISSING _ DAMAGED

NUMBER OR MONTH

Thank you. Once a clain is received and resolved, delivery of replacement issues routinely takes $4-6$ weeks.

(TO BE FILLED OUT BY APA STAFF)

DATE RECEIVED:

ACTION TAKEN:

STAFF NAME:
DATE OF ACTION:

INV. NO. \& DATE:

LABEL NO. \& DATE:

Send this form to APA Subseription Claims, 750 First Street, NE, Washington, DC 20002-4242

PLEASE DO NOT REMOVE. A PHOTOCOPY MAY BE USED. 